\title{
Frailty Models and Copulas: Similarities and Dif-
}

\section{ferences}

KLARA GOETHALS, ${ }^{*}$ PAUL JANSSEN ${ }^{* *} \&$ LUC DUCHATEAU*

*Department of Physiology and Biometrics, Ghent University, Belgium;

${ }^{* *}$ Center for Statistics, Hasselt University, Belgium

Correspondence Address: Klara Goethals, Department of Physiology and Biometrics, Faculty of Veterinary Medicine, Ghent University, Salisburylaan 133, 9820 Merelbeke, Belgium. Email: Klara.Goethals@UGent.be

ABSTRACT Copulas and frailty models are important tools to model bivariate survival data. Equivalence between Archimedean copula models and shared frailty models, e.g., between the Clayton-Oakes copula model and the shared gamma frailty model, has often been claimed in the literature. In this note we show that, in both models, there is indeed the well known equivalence between the copula functions; the modeling of the marginal survival functions, however, is quite different. The latter fact leads to different joint survival functions.

KEY WORDS: Bivariate survival data, Clayton-Oakes copula, Positive stable frailty, Shared gamma frailty model 


\section{Introduction}

Clustered survival data arise when event times belonging to the same cluster are correlated. We will consider bivariate survival data, i.e., clustered survival data with clusters of size two. To set the scene, consider the diagnosis of hip fracture being healed in a dog (Risselada et al., 2006). The time to diagnosis is assessed by two different imaging techniques, radiography (RX) and ultrasound (US) resulting in two clustered (within dog) diagnosis times.

Lee et al. (1992) and Spiekerman \& Lin (1998) study the properties of marginal Cox regression models for clustered (bivariate) survival data. They show that the estimators for the regression coefficients are consistent and they obtain an appropriate version of the asymptotic variance-covariance matrix of the estimators that takes the clustering into account.

This marginal regression model approach, however, does not give any information on the dependence between the diagnosis times in a cluster.

When the dependence itself is of interest, it needs to be modeled. The two models that have been used most frequently for bivariate survival data are copula models and frailty models.

In copula models the joint survival function of the two diagnosis times in a dog is modeled as a function, called the copula, of the marginal survival functions of the two diagnosis times (see Section 2). The copula, used to couple the marginal survival functions and the joint survival function, determines the type of dependence. In this 
note we consider parametric copula functions; the marginal survival functions can be modeled in a parametric, a semi-parametric or a nonparametric way. An excellent reference on copulas is Nelsen (2006).

Inferential procedures for copula models typically use a two-stage approach (e.g., see Shih \& Louis (1995)). In the first stage the marginal survival functions are estimated (parametric, semi-parametric or nonparametric estimation has been considered). In the second stage, estimates for the parameters in the copula function are obtained by maximization of the likelihood with respect to the copula function parameter, after we have replaced the (derivatives of the) marginal survival functions by the corresponding estimated versions (obtained in the first stage) in the likelihood expression.

When modeling the marginal survival functions in a semi-parametric or nonparametric way the two-stage approach is a natural way to arrive at sound statistical inference. For marginal survival functions modeled in a parametric way also maximum likelihood estimation for all the parameters simultaneously (i.e., the parameters of the marginal survival functions and the parameters of the copula) is possible.

The frailty model is a conditional hazard model which has a multiplicative factor, called the frailty, which models the random cluster effect. Conditional on the frailty, the diagnosis times within a cluster are assumed to be independent. Starting from the conditional hazard model, the joint survival function can be obtained by integrating out the frailty (using an appropriate frailty density and its corresponding Laplace transform) in the conditional bivariate survival distribution. The joint 
survival functions takes the form of an Archimedean copula (see Section 2). Based on this finding it is often stated that the frailty model corresponds to a particular Archimedean copula model (Manatunga \& Oakes, 1999; Viswanathan \& Manatunga, 2001; Andersen, 2005). This statement is, however, confusing since the two modeling approaches are quite different in nature.

Explaining the similarities and the differences from a modeling as well as from an inferential point of view is the main purpose of this note. In Section 2, we introduce the copula model and the frailty model for bivariate survival data in a general way. In Section 3, we compare the Clayton-Oakes copula with Weibull marginal survival functions as arguments and the shared gamma frailty model with conditional Weibull hazards and we demonstrate that the two models are of different nature. We also discuss the case where the marginal survival functions (copula models) and the conditional hazards (frailty models) are modeled in a semi-parametric and a nonparametric way.

Section 4 contains a similar comparison for the positive stable copula and the shared frailty model with positive stable frailty density. For the particular case of Weibull marginal survival functions and conditional Weibull hazards we show that the two models lead to the same inferential results. 


\section{The Copula and the Frailty Model}

Consider two clustered diagnostic times $\left(T_{1}, T_{2}\right)$ ( $T_{1}$ for RX, $T_{2}$ for US) and let $S_{1, c}(t)$ and $S_{2, c}(t)$ be the marginal survival functions for the RX and US imaging technique.

For a copula model the joint survival function is given by

$$
S_{c}\left(t_{1}, t_{2}\right)=C_{\theta}\left\{S_{1, c}\left(t_{1}\right), S_{2, c}\left(t_{2}\right)\right\}
$$

with $C_{\theta}$ a copula function, i.e., a distribution function on the unit square $C_{\theta}:[0,1]^{2} \rightarrow[0,1]:(u, v) \rightarrow C_{\theta}(u, v)$ parameterized by $\theta$ (possibly a vector).

The frailty model, on the other hand, is given by

$$
h_{i j}(t)=u_{i} h_{j, u}(t)
$$

with $h_{i j}(t)$ the hazard at time $t$ in cluster $i, i=1 \ldots, s$, for diagnosis technique $j$ $(1=\mathrm{RX}, 2=\mathrm{US}), h_{j, u}(t)$ the hazard at time $t$ for a cluster with frailty equal to one and diagnosis technique $j$ and $u_{i}$ the frailty term. The density of a frailty random variable $U_{i}$ is denoted as $f_{U}($.$) .$

To compare copula models and frailty models we need the family of Archimedean copulas

$$
C_{\theta}(u, v)=p\{q(u)+q(v)\}
$$

where $p($.$) is any nonnegative decreasing function with p(0)=1$ and nonnegative 
second derivative and $q($.$) is its inverse function; p($.$) is parametrized by \theta$.

To make the link between copula and frailty models, we only need functions $p($. that are Laplace transforms of frailty densities $f_{U}($.$) (frailty densities have support$ $[0, \infty[)$

$$
p(s)=L(s)=\mathrm{E}\{\exp (-U s)\}=\int_{0}^{\infty} \exp (-u s) f_{U}(u) d u
$$

leading to

$$
C_{\theta}(u, v)=L\left\{L^{-1}(u)+L^{-1}(v)\right\}
$$

For the copula model the joint survival function is

$$
S_{c}\left(t_{1}, t_{2}\right)=L\left[L^{-1}\left\{S_{1, c}\left(t_{1}\right)\right\}+L^{-1}\left\{S_{2, c}\left(t_{2}\right)\right\}\right] .
$$

For the frailty model the joint conditional survival function for cluster $i$ is $S_{i}\left(t_{1}, t_{2}\right)=$ $\exp \left[-u_{i}\left\{H_{1, u}\left(t_{1}\right)+H_{2, u}\left(t_{2}\right)\right\}\right]$ with $H_{j, u}(t)=\int_{0}^{t} h_{j, u}(s) d s$ the cumulative baseline hazard for diagnosis technique $j$.

The joint survival function can be obtained by integrating out the frailties with respect to the frailty density

$$
\begin{aligned}
S_{m}\left(t_{1}, t_{2}\right) & =\int_{0}^{\infty} S_{i}\left(t_{1}, t_{2}\right) f_{U}\left(u_{i}\right) d u_{i} \\
& =\mathrm{E}\left[\exp \left\{-U\left(H_{1, u}\left(t_{1}\right)+H_{2, u}\left(t_{2}\right)\right)\right\}\right] .
\end{aligned}
$$


The joint survival function derived from the frailty model (3) and the joint survival function specified for the copula model (2) are two different ways to model $\mathrm{P}\left(T_{1}>t_{1}, T_{2}>t_{2}\right)$

Expression (3) is nothing but the Laplace transform of the frailty density function evaluated at $s=H_{1, u}\left(t_{1}\right)+H_{2, u}\left(t_{2}\right)$ so that

$$
S_{m}\left(t_{1}, t_{2}\right)=L\left\{H_{1, u}\left(t_{1}\right)+H_{2, u}\left(t_{2}\right)\right\}
$$

Furthermore, the marginal survival function for each of the two imaging techniques can be obtained by putting the diagnosis time for the other diagnostic technique equal to zero in (4) and thus $S_{j, m}(t)=L\left\{H_{j, u}(t)\right\}$. It follows that

$$
H_{j, u}(t)=L^{-1}\left\{S_{j, m}(t)\right\}
$$

Using this relationship, (4) can be written as

$$
S_{m}\left(t_{1}, t_{2}\right)=L\left[L^{-1}\left\{S_{1, m}\left(t_{1}\right)\right\}+L^{-1}\left\{S_{2, m}\left(t_{2}\right)\right\}\right] .
$$

Remark that the copula used in the joint survival functions (2) and (6) is the same. The arguments of the copula, the marginal survival functions, however, are not the same. From (2) and (6) it follows that the two models are different in nature. This will be demonstrated in Section 3, where we compare the Clayton-Oakes copula with Weibull marginal survival functions as arguments and the shared gamma frailty model with conditional Weibull hazards. A similar comparison, in Section 4, for 
the positive stable copula and the shared frailty model with positive stable frailty density, shows the exceptional character of this model, in the sense that both models are equivalent.

\section{The Clayton-Oakes Copula and the Gamma Frailty}

\section{Model}

In the copula model assume that the marginal survival functions are obtained from Weibull hazards and use the two-stage approach of Shih \& Louis (1995). In the first step, parameter estimates for $\lambda_{j}$ and $\rho_{j}$ are obtained by fitting the following survival model in each group (RX or US) separately

$$
h_{j, c}(t)=\lambda_{j} \rho_{j} t^{\rho_{j}-1}
$$

with $j=1$ for the RX diagnosis and $j=2$ for the US diagnosis, i.e., $S_{j, c}(t)=$ $\exp \left(-\lambda_{j} t^{\rho_{j}}\right)$

As an example, we use time to diagnosis data based on RX and US from 106 dogs. The parameter estimates (ML estimates) for our example are $\hat{\lambda}_{1}=0.106, \hat{\rho}_{1}=2.539$, $\hat{\lambda}_{2}=0.219$ and $\hat{\rho}_{2}=2.323$. To model the dependence we use the joint survival function (2) with

$$
L(s)=(1+\theta s)^{-1 / \theta} \text { and } L^{-1}(s)=\left(s^{-\theta}-1\right) / \theta
$$


with $\theta \geq 0 . L($.$) is the Laplace transform of the one-parameter gamma density (see$ (11)). The corresponding copula $C_{\theta}(u, v)=\left(u^{-\theta}+v^{-\theta}-1\right)^{-1 / \theta}$ is the ClaytonOakes copula.

The joint survival function then becomes

$$
S_{c}\left(t_{1}, t_{2}\right)=\left[\left\{S_{1, c}\left(t_{1}\right)\right\}^{-\theta}+\left\{S_{2, c}\left(t_{2}\right)\right\}^{-\theta}-1\right]^{-1 / \theta}
$$

Based on the joint survival function (9) the likelihood can be constructed (see e.g. Shih \& Louis (1995)). In the second step we replace in the likelihood $S_{j, c}($.$) by$ $\hat{S}_{j, c}($.$) , obtained by replacing \lambda_{j}, \rho_{j}$ by $\hat{\lambda}_{j}, \hat{\rho}_{j}$ (for $\left.j=1,2\right)$, and we then maximize the likelihood with respect to $\theta$. In our example $\hat{\theta}$ is 0.890 .

Since the marginal survival functions and the copula are modeled in a parametric way, the likelihood obtained from the joint survival function can also be maximised jointly for the marginal survival function parameters and the copula function parameter, leading to parameter estimates $\hat{\lambda}_{1}=0.145, \hat{\rho}_{1}=2.341, \hat{\lambda}_{2}=0.233, \hat{\rho}_{2}=2.212$ and $\hat{\theta}=1.066$. Durrleman et al. (2000) give a detailed comparison between the two-stage approach and the (joint) maximization of the likelihood.

For the frailty model we start from a conditional Weibull hazard with different $\tilde{\lambda}$ and $\widetilde{\rho}$ parameters for the two diagnostic techniques (this is similar to the way in which the marginal survival functions in the copula model were modeled)

$$
h_{i j}(t)=u_{i} \widetilde{\lambda}_{j} \widetilde{\rho}_{j} t^{\widetilde{\rho}_{j}-1}
$$


with $u_{1}, \ldots, u_{s}$ independent realizations of a one parameter gamma density with mean one and variance $\theta$

$$
f_{U}\left(u_{i}\right)=\frac{u_{i}^{\frac{1}{\theta}-1} \exp \left(\frac{-u_{i}}{\theta}\right)}{\theta^{\frac{1}{\theta}} \Gamma\left(\frac{1}{\theta}\right)} .
$$

The Laplace transform for the gamma distribution and its inverse is given in (8). Plugging in (8) into (4) leads to the joint survival function

$$
S_{m}\left(t_{1}, t_{2}\right)=\left[1+\theta\left\{H_{1, u}\left(t_{1}\right)+H_{2, u}\left(t_{2}\right)\right\}\right]^{-1 / \theta} .
$$

Making use of (5) this can be rewritten as

$$
\begin{aligned}
S_{m}\left(t_{1}, t_{2}\right) & =\left[1+\left[\left\{S_{1, m}\left(t_{1}\right)\right\}^{-\theta}-1\right]+\left[\left\{S_{2, m}\left(t_{2}\right)\right\}^{-\theta}-1\right]\right]^{-1 / \theta} \\
& =\left[\left\{S_{1, m}\left(t_{1}\right)\right\}^{-\theta}+\left\{S_{2, m}\left(t_{2}\right)\right\}^{-\theta}-1\right]^{-1 / \theta}
\end{aligned}
$$

This expression looks similar to the copula form representation in (9). There is, however, the substantial difference that $S_{j, m}(t) \neq S_{j, c}(t), j=1,2$. The marginal survival function $S_{j, m}(t)=\left(1+\theta \widetilde{\lambda}_{j}{\widetilde{\tau^{j}}}^{-1 / \theta}\right.$ is not of the Weibull form. Note that the copula parameter also shows up in $S_{j, m}($.$) .$

Parameter estimates for shared frailty models with a parametric baseline function can be easily obtained through maximization of the observable likelihood (Klein, 1992; Costigan \& Klein, 1993). Estimates for the parameters $\widetilde{\lambda}_{1}, \widetilde{\rho}_{1}, \widetilde{\lambda}_{2}, \widetilde{\rho}_{2}$ and $\theta$ of the frailty model are given by $0.079,3.827,0.218,3.456$ and 0.909 respectively. 
The parameter estimates for $\widetilde{\lambda}_{j}, \widetilde{\rho}_{j}$ from the frailty model have a conditional meaning (conditional on the particular cluster) whereas the parameter estimates from the copula model refer to the marginal hazard and survival functions. To compare the two models, note that the marginal hazard function in the frailty model is given by

$$
h_{j, m}(t)=\widetilde{\lambda}_{j} \widetilde{\rho}_{j} t^{\widetilde{\rho}_{j}-1}\left(1+\theta \widetilde{\lambda}_{j}{\widetilde{\rho^{j}}}^{-1}\right.
$$

whereas the marginal hazard in the copula model is given by (7) and is free of $\theta$. Figure 1 shows the marginal hazards in the copula model and in the frailty model, the pictures use the estimated parameters.

For $\widetilde{\rho}_{j}>1$, as is the case in our example, the conditional hazard in (10) is a monotone increasing function. With $\widetilde{\rho}_{j}>1$ the marginal hazard function (12) reaches a maximum in $t=\left\{\left(\widetilde{\rho}_{j}-1\right) /\left(\theta \widetilde{\lambda}_{j}\right)\right\}^{1 / \widetilde{\rho}_{j}}$. The marginal hazard in the copula model is monotone increasing. Therefore the marginal hazard functions in the two models can never be the same.

Figure 1

We also fitted the copula and frailty model using the semi-parametric (Cox) model and the nonparametric model for the (conditional) hazards.

The semi-parametric copula model is given by 


$$
h_{j, c}(t)= \begin{cases}h_{0}(t) & \text { for RX } \\ h_{0}(t) \exp (\beta) & \text { for US }\end{cases}
$$

and the nonparametric copula model by

$$
h_{j, c}(t)= \begin{cases}h_{1}(t) & \text { for RX } \\ h_{2}(t) & \text { for US }\end{cases}
$$

with $h_{0}(t), h_{1}(t)$ and $h_{2}(t)$ unspecified hazard functions.

Estimation for the semi-parametric and nonparametric copula model is typically based on the two-stage approach (Shih \& Louis, 1995; Spiekerman \& Lin, 1998; Andersen, 2005). For the semi-parametric model, we obtain in the first stage an estimate of $\beta$ through partial likelihood maximisation and we use the Breslow estimator of $S_{0}(t)=\exp \left(-\int_{0}^{t} h_{0}(t)\right)$ (Breslow, 1974). For the nonparametric approach, we use the Nelson-Aalen estimator of $S_{j, c}(t)=\exp \left(-\int_{0}^{t} h_{j}(t)\right), j=1,2$ (Nelson, 1972; Aalen, 1978). In the second stage we replace the marginal survival functions in the likelihood by their corresponding estimates and then maximize with respect to $\theta$.

The semi-parametric frailty model is given by

$$
h_{i j}(t)= \begin{cases}u_{i} \widetilde{h}_{0}(t) & \text { for RX } \\ u_{i} \widetilde{h}_{0}(t) \exp (\widetilde{\beta}) & \text { for US }\end{cases}
$$

and the nonparametric frailty model by 


$$
h_{i j}(t)=\left\{\begin{array}{cc}
u_{i} \widetilde{h}_{1}(t) & \text { for RX } \\
u_{i} \widetilde{h}_{2}(t) & \text { for US }
\end{array}\right.
$$

with again $\widetilde{h}_{0}(t), \widetilde{h}_{1}(t)$ and $\widetilde{h}_{2}(t)$ unspecified hazard functions.

Estimation for the semi-parametric frailty model is based on the EM-algorithm (Klein, 1992). Estimation for the nonparametric frailty model is also based on the EM-algorithm but introducing imaging technique as stratification factor.

Parameter estimates in the semi-parametric copula model are $\hat{\beta}=0.508$ and $\hat{\theta}=$ 0.997; in the semi-parametric (Cox) gamma frailty model estimates are given by $\hat{\beta}=0.828$ and $\hat{\theta}=1.250$. In the nonparametric copula approach the estimate for $\theta$ is 1.236; in the nonparametric gamma frailty model it is 1.204 .

\section{The Positive Stable Copula and Frailty Model}

In the two-stage copula approach, the marginal survival functions corresponding to (7) are used, but the copula function now uses the Laplace transform

$$
L(s)=\exp \left(-s^{\theta}\right) \text { and } L^{-1}(s)=(-\log s)^{1 / \theta}
$$

with $0 \leq \theta<1$. $L($.) is the Laplace transform of the positive stable density (see (15)). The corresponding copula takes form 


$$
C_{\theta}(u, v)=\exp \left[-\left\{(-\log u)^{1 / \theta}+(-\log v)^{1 / \theta}\right\}^{\theta}\right]
$$

Therefore the joint survival function in the positive stable copula model is

$$
S_{c}\left(t_{1}, t_{2}\right)=\exp \left[-\left[\left\{-\log S_{1, c}\left(t_{1}\right)\right\}^{1 / \theta}+\left\{-\log S_{2, c}\left(t_{2}\right)\right\}^{1 / \theta}\right]^{\theta}\right]
$$

The parameter estimates $\hat{\lambda}_{1}, \hat{\rho}_{1}, \hat{\lambda}_{2}$ and $\hat{\rho}_{2}$ are obviously the same as for the ClaytonOakes copula model. As we did for the Clayton-Oakes copula, we replace the $S_{j, c}($.$) 's$ in the (joint survival functions appearing in the) likelihood and we maximize with respect to $\theta$.

Under this new dependency structure the value of the association parameter $\theta$ is estimated as 0.563 .

Since the marginal survival functions and the copula are modeled in a parametric way, the likelihood can be maximized jointly for the marginal survival function parameters and the copula function parameter. The estimates obtained from this approach are shown in Table 1.

In the frailty model approach, we fit the conditional model (10) to the data, with the positive stable density as frailty density (Hougaard, 1986)

$$
f_{U}\left(u_{i}\right)=-\frac{1}{\pi u_{i}} \sum_{k=1}^{\infty} \frac{\Gamma(k \theta+1)}{k !}\left(-u_{i}^{-\theta}\right)^{k} \sin (\theta k \pi)
$$

with $0 \leq \theta<1$. 
This complex expression for the positive stable density translates into the simple Laplace transform (13).

From (4) the joint survival function is

$$
S_{m}\left(t_{1}, t_{2}\right)=\exp \left[-\left\{H_{1, u}\left(t_{1}\right)+H_{2, u}\left(t_{2}\right)\right\}^{\theta}\right]
$$

Making use of (5) and (13) this can be rewritten as

$$
S_{m}\left(t_{1}, t_{2}\right)=\exp \left[-\left[\left\{-\log S_{1, m}\left(t_{1}\right)\right\}^{1 / \theta}+\left\{-\log S_{2, m}\left(t_{2}\right)\right\}^{1 / \theta}\right]^{\theta}\right]
$$

which has the same form as (14).

Also for the shared frailty model with positive stable frailty density, the frailties can be integrated out to obtain the observable likelihood which can then be maximized with respect to all the parameters (Costigan \& Klein, 1993).

Parameter estimates for $\widetilde{\lambda}_{1}, \widetilde{\rho}_{1}, \widetilde{\lambda}_{2}, \widetilde{\rho}_{2}$ and $\widetilde{\theta}$ are provided in Table 1.

For the positive stable copula with marginal Weibull hazards we have $S_{j, c}(t)=$ $\exp \left(-\lambda_{j} t^{\rho_{j}}\right)$; for the shared positive stable frailty model with conditional Weibull hazards $S_{j, m}(t)=\exp \left(-\widetilde{\lambda}_{j}^{\theta} \widetilde{t}^{\widetilde{\rho}_{j}} \theta\right)$

So in both models the marginal survival functions are Weibull, i.e., the event times are Weibull distributed. We can make the Weibull distributions identical by taking

$$
\lambda_{j}=\widetilde{\lambda}_{j}^{\theta} \quad \rho_{j}=\theta \widetilde{\rho}_{j}
$$

Assuming bivariate survival data without censoring, the likelihood (which is the 
product over the clusters of the bivariate densities) for the copula function is

$$
L_{c}=\prod_{i=1}^{s} \exp \left(-z_{i}^{\theta}\right) \lambda_{1} \rho_{1} t_{i 1}^{\rho_{1}-1} \lambda_{2} \rho_{2} t_{i 2}^{\rho_{2}-1}\left\{\frac{z_{i}^{2(\theta-1)}+(1 / \theta-1) z_{i}^{\theta-2}}{\left(z_{i 1} z_{i 2}\right)^{(\theta-1)}}\right\}
$$

with $z_{i j}=\left(\lambda_{j} t_{i j}^{\rho_{j}}\right)^{1 / \theta}$ and $z_{i}=z_{i 1}+z_{i 2}$.

For the frailty model the observable likelihood is

$$
L_{m}=\prod_{i=1}^{s} \exp \left(-\widetilde{z}_{i}^{\theta}\right) \widetilde{\lambda}_{1} \widetilde{\rho}_{1} t_{i 1}^{\widetilde{\rho}_{1}-1} \widetilde{\lambda}_{2} \widetilde{\rho}_{2} t_{i 2}^{\widetilde{\rho}_{2}-1}\left\{\theta^{2} \widetilde{z}_{i}^{2(\theta-1)}+\theta(1-\theta) \widetilde{z}_{i}^{\theta-2}\right\}
$$

with $\widetilde{z}_{i j}=\left(\widetilde{\lambda}_{j} t_{i j}^{\widetilde{\rho}_{j}}\right)$ and $\widetilde{z}_{i}=\widetilde{z}_{i 1}+\widetilde{z}_{i 2}$

From (17) we easily see that

$$
\widetilde{z}_{i j}=z_{i j}
$$

Using (17) and (18) one can show that $L_{m}$ can be rewritten as $L_{c}$. As an illustration check in Table 1 that for the estimates obtained from the maximization (jointly for all the parameters) of $L_{m}$, resp. maximization of $L_{c}$, the relations (17) hold.

The fact that the parameters of the copula and frailty model can be identified, as discussed in (17), seems to be an exclusive property of the combination of Weibull distributed event times and frailties from a positive stable distribution. If the exponential distribution is assumed for the event times together with a positive stable distribution for the frailties for example, this property vanishes. Under these assumptions the population hazard function in the copula model $h_{j, c}(t)=\lambda_{j}$ is constant, but in the frailty model the marginal hazard function is no longer constant, 
but Weibull: $h_{j, m}(t)=\theta \widetilde{\lambda}_{j}^{\theta} t^{\theta-1}$.

\section{Conclusions}

In this paper we discussed similarities and differences between copula models and frailty models for bivariate survival data. We focused on the comparison between the Clayton-Oakes copula model and the shared gamma frailty model; and between the positive stable copula model and the shared positive stable frailty model. For each of the two comparisons, the copula functions used for the bivariate joint survival functions are the same but the marginal survival functions are modeled in a different way. To show the differences in a concrete example, we use the Clayton-Oakes copula model with Weibull marginal survival functions and the shared gamma frailty model with conditional Weibull survival functions (Section 3). A similar comparison between the positive stable copula model and the shared positive stable copula model shows that, in the exceptional case of the Weibull, there is a one-to-one match between the two models (Section 4).

With the more flexible semi-parametric and nonparametric model specification, parameter estimates of the copula model are typically obtained by separate modeling of the marginal survival functions (step 1) and the copula function (step 2). Therefore there is complete separation between the estimation of the marginal survival function parameters and the copula function parameter. This provides an explanation why the estimate of the copula function parameter in the copula and frailty 
model approach differs.

\section{Acknowledgements}

The authors thank both referees for their insightful remarks, one of them pointed out an inaccuracy in the first version of this paper. The first author acknowledges the financial support of the Research Fund of Ghent University, Ghent, Belgium (grant no. B/04953/01). The research of Luc Duchateau and Paul Janssen was supported by IAP research network grant nr. P6/03 of the Belgian government (Belgian Science Policy).

\section{References}

Aalen, O.O. (1978) Nonparametric inference for a family of counting processes, Annals of Statistics, 6(4), pp. 701-726.

Andersen, E.W. (2005) Two-stage estimation in copula models used in family studies, Lifetime Data Analysis, 11(3), pp. 333-350.

Breslow, N.E. (1974) Covariance analysis of censored survival data, Biometrics, 30(1), pp. 89-99.

Costigan, M. \& Klein, J.P. (1993) Multivariate survival analysis based on frailty models, in: A.P. Basu (Ed.) Advances in Reliability, pp. 43-58 (North Holland). 
Cox, D.R. (1972) Regression models and life-tables, Journal of the Royal Statistical Society. Series B (Methodological), 34(2), pp. 187-220.

Durrleman, V., Nikeghbali, A. \& Roncalli, T. (2000) Which copula is the right one?, Technical Report. Groupe de Recherche Opérationnelle, Crédit Lyonnais, France.

Glidden, D.V. (2000) A two-stage estimator of the dependence parameter for the Clayton-Oakes model, Lifetime Data Analysis, 6(2), pp. 141-156.

Hougaard, P. (1986) Survival models for heterogeneous populations derived from stable distributions, Biometrika, 73(2), pp. 387-396.

Klein, J.P. (1992) Semiparametric estimation of random effects using the Cox model based on the EM algorithm, Biometrics, 48(3), pp. 795-806.

Lee, E.W., Wei, L.J. \& Amato, D.A. (1992) Cox-type regression analysis for large numbers of small groups of correlated failure time observations, in: J. Klein and P. Goel (Eds.) Survival Analysis: State of the Art, pp. 237-247 (Kluwer and Dordrecht).

Manatunga, A.K. \& Oakes, D. (1999) Parametric analysis for matched pair survival data, Lifetime Data Analysis, 5(4), pp. 371-387.

Nelsen, R.B. (2006) An introduction to copulas (2nd ed.) (New York: Springer Verlag). 
Nelson, W. (1972) Theory and applications of hazard plotting for censored failure data, Technometrics 14(4), pp. 945-965.

Risselada, M., van Bree, H., Kramer, M., Chiers, K., Duchateau, L., Verleyen, P. \& Saunders, J.H. (2006) Evaluation of nonunion fractures in dogs by use of B-mode ultrasonography, power Doppler ultrasonography, radiography, and histologic examination, American Journal of Veterinary Research 67(8), pp. 1354-1361.

Shih, J.H. \& Louis, T.A. (1995) Inferences on the association parameter in copula models for bivariate survival data, Biometrics 51(4), pp. 1384-1399.

Spiekerman, C.F. \& Lin, D.Y. (1998) Marginal regression models for multivariate failure time data, Journal of the American Statistical Association 93(443), pp. $1164-1175$.

Viswanathan, B. \& Manatunga, A.K. (2001) Diagnostic plots for assessing the frailty distribution in multivariate survival data, Lifetime Data Analysis 7(2), pp. 143155. 
Table 1: Estimates of the copula function parameter and the Weibull parameters of the positive stable copula model (using the two-stage approach or joint estimation) and of the positive stable frailty model.

Figure 1: The marginal hazard functions from the frailty model and the copula model with gamma frailty density for the time to diagnosis of being healed data assessed by either US or RX. 
Table 1:

Frailty model

Copula model

\begin{tabular}{ccccc} 
Parameter & & Parameter & two-stage & joint estimation \\
\hline$\widetilde{\lambda}_{R X}$ & 0.020 & $\lambda_{R X}$ & 0.106 & 0.118 \\
$\tilde{\rho}_{R X}$ & 4.560 & $\rho_{R X}$ & 2.539 & 2.491 \\
$\widetilde{\lambda}_{U S}$ & 0.059 & $\lambda_{U S}$ & 0.219 & 0.213 \\
$\widetilde{\rho}_{U S}$ & 4.240 & $\rho_{U S}$ & 2.323 & 2.315 \\
$\widetilde{\theta}$ & 0.546 & $\theta$ & 0.563 & 0.546 \\
\hline \hline
\end{tabular}


Figure 1:

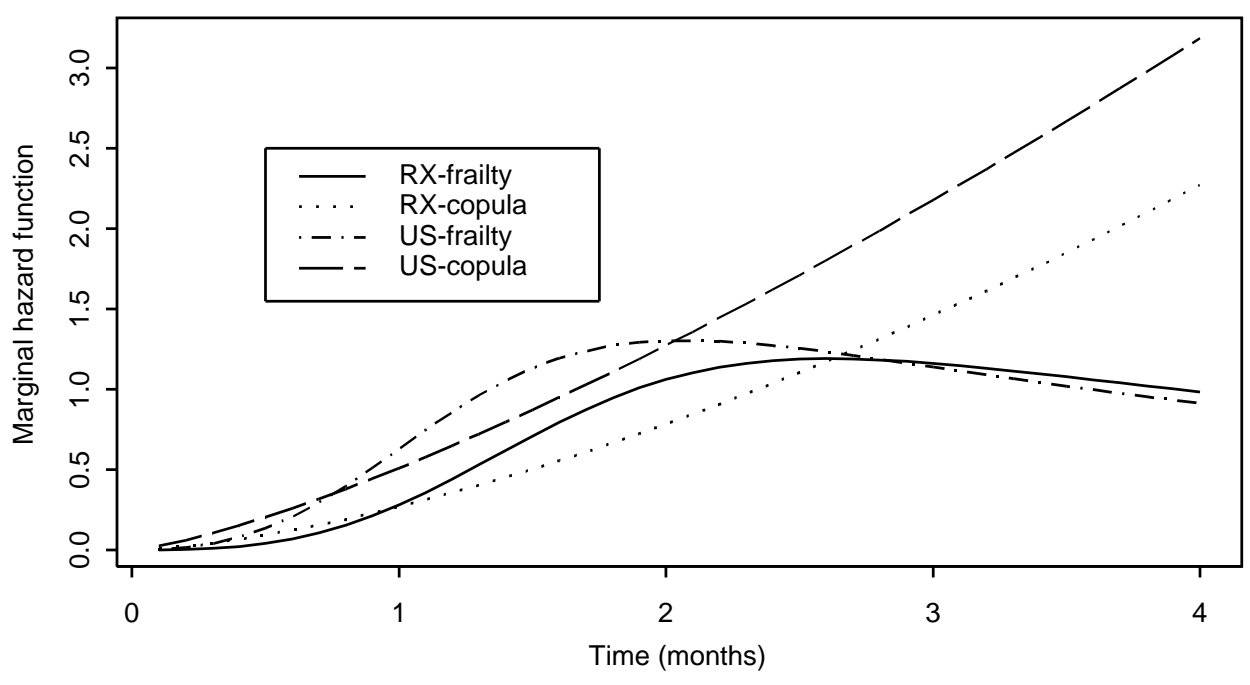

